\title{
A fortuna crítica (acadêmica) de Lúcio Cardoso
}

Ésio Macedo Ribeiro

Universidade de São Paulo

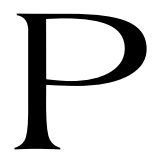

assados quatro anos da morte de Lúcio Cardoso, no ano de 1972, era apresentado na Universidade de São Paulo o primeiro trabalho voltado ao estudo da sua obra. De lá para cá, até o início de 2008, mais 67 se somaram a este, entre dissertações de mestrado e teses de doutorado, em torno à vida e à obra de Lúcio Cardoso.

Este artigo visa a proporcionar ao estudioso de Letras o levantamento exaustivo dos textos acadêmicos, realizados no Brasil e no exterior, acerca do escritor Lúcio Cardoso. Embora nem todos os 68 trabalhos abordem em sua totalidade a obra cardosiana, julguei conveniente inseri-los, porque em pesquisa nenhuma fonte deve ser desprezada, por menor que seja.

A seguir, a bibliografia levantada até o primeiro semestre de 2008:

ALBERGARIA, Maria Consuelo de Pádua. O espaço da loucura em Minas Gerais - análise de ficção de Cornélio Pena. 1982. 320 p. Tese (Doutorado em Letras) Faculdade de Letras, Universidade Federal do Rio de Janeiro, Rio de Janeiro, 1982.

ALMEIDA, Raquel Cestaro Freesz de. Crônica da casa assassinada, Nina: o resgate de uma mulher desejante. 2004.87 p. Dissertação (Mestrado em Letras) - Centro de Ensino Superior de Juiz de Fora, Juiz de Fora - MG, 2004.

ARAÚJO, Francisco Sérgio Souza de. As memórias que falam em Crônica da casa assassinada. 2005. 125 p. Dissertação (Mestrado em Letras) - Universidade Federal do Ceará, Fortaleza, 2005.

ARAÚJO, Márcia Maria de Melo. Os abismos internos das personagens e os espaços degradados na trilogia "O mundo sem Deus", de Lúcio Cardoso. 2007. 120 p. Dissertação (Mestrado em Literatura) - Faculdade de Letras, Universidade Federal de Goiás, Goiânia, 2007. 
ARCO E FLEXA, Teresinha de Almeida. Lúcio Cardoso e Julien Green: transgressão e culpa. 1990.2 v. 378 p. Tese (Doutorado em Língua e Literatura Francesa) - Faculdade de Filosofia, Letras e Ciências Humanas, Universidade de São Paulo, São Paulo, 1990. ${ }^{1}$

BARBOSA, Kristianny Brandão. Imagens do feminino: um estudo comparativo em "A casa de Bernarda Alba", de Federico García Lorca e "O escravo", de Lúcio Cardoso. 2005. 107 p. Dissertação (Mestrado em Letras e Lingüística) - Centro de Ciências Humanas, Letras e Artes, Universidade Federal de Alagoas, Maceió, 2005.

BARROS JÚNIOR, Fernando Monteiro de. Aristocracia e monstruosidade: nostalgia da aura na Crônica da casa assassinada de Lúcio Cardoso. 1994.131 p. Dissertação (Mestrado em Literatura Brasileira) - Faculdade de Letras, Universidade Federal do Rio de Janeiro, Rio de Janeiro, 1994.

. Vampiros na casa-grande: clausura eposes do gótico em Lúcio Cardoso. 2002. 168 p. Tese (Doutorado em Literatura Brasileira) - Faculdade de Letras, Universidade Federal do Rio de Janeiro, Rio de Janeiro, 2002.

BARROS, José Américo de Miranda. A constituição do narrador na ficção de Lúcio Cardoso. 1987.218 p. Dissertação (Mestrado em Literatura Brasileira) Faculdade de Letras, Universidade Federal de Minas Gerais, Belo Horizonte, 1987.

BARROS, Marta Cavalcante de. Espaço e decadência na Crônica da casa assassinada de Lúcio Cardoso. 1997. 148 p. Dissertação (Mestrado em Letras - Teoria Literária e Literatura Comparada) - Faculdade de Filosofia, Letras e Ciências Humanas, Universidade de São Paulo, São Paulo, 1997.

BASSANI, Geraldo. Caligrafia das sombras: vozes, versões - visitando a Crônica da casa assassinada. 2000. 125 p. Dissertação (Mestrado em Letras) - Universidade Federal do Espírito Santo, Vitória, ES, 2000.

BEDRAN, Ângela Maria. Nacos de real - a paixão da escrita em Lúcio Cardoso e Clarice Lispector. 2000. 125 p. Dissertação (Mestrado em Letras - Estudos Literários) - Faculdade de Letras, Universidade Federal de Minas Gerais, Belo Horizonte, 2000 .

\footnotetext{
${ }^{1}$ Este trabalho encontra-se no prelo e será publicado brevemente pela Editora da Universidade de São Paulo. A professora, atualmente, assina seus textos desse modo: Teresa de Almeida.
} 
BONAPACE, Adolphina Portella. O belo posto em questão: Crônica da casa assassinada de Lúcio Cardoso. 1980. 302 p. Tese (Doutorado em Letras) Faculdade de Letras, Universidade Federal do Rio de Janeiro, Rio de Janeiro, 1980.

BRAGA FILHO, Jair Ramos. A melancolia narrada: Dias perdidos de Lúcio Cardoso. 2008. 131 p. Dissertação (Mestrado em Letras - Estudos Literários) - Setor de Ciências Humanas, Letras e Artes, Universidade Federal do Paraná, Curitiba, 2008.

BRANDÃO, Ruth Silviano. Mulher ao pé da letra: a personagem feminina na literatura. 1989. 343 p. Tese (Doutorado em Letras) - Faculdade de Letras, Universidade Federal de Minas Gerais, Belo Horizonte, 1989. ${ }^{2}$

BUENO, Ana Alice. A gramática do descontínuo - uma leitura de Inácio, de Lúcio Cardoso. 2007, 100 p. Dissertação (Mestrado em Teoria Literária) - Universidade Federal de Santa Catarina, Florianópolis, 2007.

CARELLI, Mario. L'univers romanesque de Lúcio Cardoso (1912-1968). 1986. 3 v., 1152 p. Tese (Doctorat d'Etat) - Universidade de Paris III - Nova Sorbonne, Paris, 1986.

CORDEIRO, Ana Maria. O escritor "Post-modernista" enquanto crítico: Lúcio Cardoso. 1995. 179 p. Dissertação (Mestrado em Literatura) - Universidade Federal de Santa Catarina, Florianópolis, 1995.

COSTA, Leda Maria da. O invisível refletido. A representação da morte e dos mortos nas memórias póstumas de Brás Cubas e Crônica da casa assassinada. 2003. 79 p. Dissertação (Mestrado em Letras) - Faculdade de Letras, Universidade do Estado do Rio de Janeiro, Rio de Janeiro, 2003.

COUTINHO, Luiz Edmundo Bouças. $O$ depoimento da errância: a experiência da palavra entre provisório e o permanente. 1978.96 p. Dissertação (Mestrado em Poética) - Faculdade de Letras, Universidade Federal do Rio de Janeiro, Rio de Janeiro, 1978.

. O desastre da imortalidade e a crônica do sujeito na poética do Diário. 1985.

174 p. Tese (Doutorado em Poética) - Faculdade de Letras, Universidade Federal do Rio de Janeiro, Rio de Janeiro, 1985.

${ }^{2} \mathrm{O}$ capítulo 5: "O discurso da morte encenada" é dedicado à análise da personagem Nina, do romance Crônica da casa assassinada. 
DIAS, Nathalia Saliba. A construção da casa assassinada. 2006. 2 v., 150 p. Dissertação (Mestrado em Letras - Estudos Literários) - Setor de Ciências Humanas, Letras e Artes, Universidade Federal do Paraná, Curitiba, 2006.

FANINI, Michele Asmar. As modulações da voz feminina na produção de Clarice Lispector: uma sociologia de sua escrita. 2004. 261 p. Dissertação (Mestrado em Sociologia) - Faculdade de Filosofia, Letras e Ciências Humanas, Universidade de São Paulo, São Paulo, 2004. ${ }^{3}$

FARIA, Viviane Fleury de. Os escombros e as formas: uma leitura de Crônica da casa assassinada, de Lúcio Cardoso, e de Lavoura arcaica, de Raduan Nassar. 2000. 134 p. Dissertação (Mestrado em Letras) - Faculdade de Letras, Universidade Federal de Goiás, Goiânia, 2000.

FORTES, Rita das Graças Félix. Tempo, espaço e decadência: uma leitura de "O som e a fúria", "Angústia", "Fogo morto" e "Crônica da casa assassinada". 2001. 344 p. Tese (Doutorado em Letras) - Universidade Federal do Rio Grande do Sul, Porto Alegre, 2001.

FRAGA, Maria Cristina Prates. Fiç̧ôes mineiras: o espaço do subsolo. 2000.190 p. Tese (Doutorado em Letras - Letras Vernáculas) - Faculdade de Letras, Universidade Federal do Rio de Janeiro, Rio de Janeiro, 2000.

FRANCISCO, Amanda Ramos. Traduzindo Moll Flanders: as versões de Lúcio Cardoso e Antonio Alves Curi sob a perspectiva dos estudos de tradução. 2003. 137 p. Dissertação (Mestrado em Letras) - Universidade Federal do Rio Grande do Sul, Porto Alegre, 2003.

FRANZ, Marcelo. Investigação do pesadelo, uma leitura em torno da Crônica da casa assassinada. 1997. 243 p. Dissertação (Mestrado em Letras - Estudos Literários) - Setor de Ciências Humanas, Letras e Artes, Universidade Federal do Paraná, Curitiba, 1997.

GUIMARÃES, Adriana Saldanha. Passeio literário pelo território das escrituras de mim - as cartas de Clarice Lispector e Lúcio Cardoso. 1998. 115 p. Dissertação (Mestrado em Letras) - Departamento de Letras e Artes, Pontifícia Universidade Católica do Rio de Janeiro, Rio de Janeiro, 1998.

${ }^{3}$ Lúcio Cardoso é citado várias vezes nessa dissertação. 
HERBOLD, Hildegard. O sagrado e o profano na literatura intimista dos anos 1930/ 1940 no Brasil: o exemplo de Lúcio Cardoso e Cornélio Pena. 1993. 120 p. Dissertação (Mestrado em História Social) - Faculdade de Filosofia, Letras e Ciências Humanas, Universidade de São Paulo, São Paulo, 1993.

JACINTHO, Valéria Franco. Cartas a Clarice Lispector - correspondência passiva da escritora depositada na Fundação Casa de Rui Barbosa. 1997. 689 p. Dissertação (Mestrado em Literatura Brasileira) - Faculdade de Filosofia, Letras e Ciências Humanas, Universidade de São Paulo, São Paulo, 1997. (Lúcio Cardoso, p. 124, 148-153, 651-675).

LOREDO NETA, Maria Madalena. Crônica da casa assassinada: uma poética da finitude. 2007. 112 p. Dissertação (Mestrado em Letras - Literatura Brasileira) - Faculdade de Letras, Universidade Federal de Minas Gerais, Belo Horizonte, 2007.

MARTINS, Vitor Hugo Fernandes. O impressionismo literário em Crônica da casa assassinada. 2003. 321 p. Tese (Doutorado em Letras) - Universidade Estadual Paulista Júlio de Mesquita Filho, UNESP, São José do Rio Preto, 2003.

MEDEIROS, Luciana Kühn Viégas de. Lições de pesquisa literária - o legado crítico de Lucia Miguel Pereira. 2005. 108 p. Dissertação (Mestrado em Ciência da Literatura) - Faculdade de Letras, Universidade Federal do Rio de Janeiro, Rio de Janeiro, 2005. ${ }^{4}$

MOREIRA, Douglas Carlos de Paula. O sentimento trágico em Crônica da casa assassinada, de Lúcio Cardoso. 2003. 139 p. Dissertação (Mestrado em Letras) - Universidade Federal do Ceará, Fortaleza, 2003.

MOURA, Waldevira Bueno Pires de. Lúcio Cardoso o narrador trágico à luz do diabo. 1992. 309 p. Dissertação (Mestrado em Letras) - Faculdade de Letras, Universidade Federal de Goiás, Goiânia, 1992.

NASCIMENTO, Maria Teresinha Martins do. Luz e sombra em Lúcio Cardoso. 1991. 244 p. Tese (Doutorado em Literatura Brasileira) - Faculdade de Letras, Universidade Federal do Rio de Janeiro, Rio de Janeiro, 1991.

\footnotetext{
${ }^{4}$ Essa dissertação reúne e analisa cerca de oitenta artigos de Lúcia Miguel Pereira, que versam sobre o romance de 30, publicados em periódicos cariocas entre 1931 e 1943 sobre a obra de, entre outros, Jorge Amado, José Américo de Almeida, Graciliano Ramos, Erico Verissimo e a prosa intimista de Lúcio Cardoso.
} 
NEVES, Júnia Nogueira. Dramas da clausura: a literatura dramática de Lúcio Cardoso. 2007. 176 p. Tese (Doutorado em Ciência da Literatura - Teoria Literária) - Universidade Federal do Rio de Janeiro, Rio de Janeiro, 2007.

OLIVEIRA, Hercules Alberto de. A epifania da verdade: morte, (homo)erotismo e espiritualidade em Crônica da casa assassinada e Grande sertão:veredas. 2005. 266 p. Tese (Doutorado em Literatura Comparada) - Faculdade de Letras, Universidade Federal Fluminense, Niterói, RJ, 2005.

PETRILLO, Regina Célia Pentagna. Signos em trânsito: o roteiro de uma buscaa poética de Lúcio Cardoso. 2002. 337 p. Tese (Doutorado em Letras - Ciências da Literatura) - Faculdade de Letras, Universidade Federal do Rio de Janeiro, Rio de Janeiro, 2002.

QUARESMA, Paulo Sérgio Andrade. A morte, os mortos e o morrer na Crônica da casa assassinada de Lúcio Cardoso. 2007. 245 p. Dissertação (Mestrado em História da Literatura) - Fundação Universidade Federal do Rio Grande, Rio Grande, RS, 2007.

RANGEL, Egon de Oliveira. Sexualidade e discurso: o verbo feito carne. 1994. 281 p. Dissertação (Mestrado em Lingüística) - Instituto de Estudos da Linguagem, Universidade Estadual de Campinas, Campinas, 1994.

RAYMUNDO, Marie Louise Hurel. Desejo e perversão: ou os atalhos da loucura (a propósito do romance Crônica da casa assassinada de Lúcio Cardoso). 1974.188 p. Dissertação (Mestrado em Literatura Brasileira) - Departamento de Letras e Artes, Pontifícia Universidade Católica do Rio de Janeiro, Rio de Janeiro, 1974.

REIS, Nelson Ricardo Guedes. A estética expressionista na obra de Lúcio Cardoso: uma luz no subsolo da casa assassinada. 2002. 221 p. Dissertação (Mestrado em Estudos Literários) - Faculdade de Letras, Universidade Federal de Minas Gerais, Belo Horizonte, 2002.

RIBEIRO, Ésio Macedo. O riso escuro ou o pavão de luto - um percurso pela poesia de Lúcio Cardoso. 2001. 285 p. Dissertação (Mestrado em Letras - Teoria Literária e Literatura Comparada) - Faculdade de Filosofia, Letras e Ciências Humanas, Universidade de São Paulo, São Paulo, 2001.

Edição crítica da poesia reunida de Lúcio Cardoso. 2006. 2 v., 1.055 p. Tese (Doutorado em Letras - Literatura Brasileira) - Faculdade de Filosofia, Letras e Ciências Humanas, Universidade de São Paulo, São Paulo, 2006. ${ }^{5}$

\footnotetext{
${ }^{5}$ A edição crítica da Poesia completa de Lúcio Cardoso encontra-se no prelo e será publicada brevemente pela Editora da Universidade de São Paulo.
} 
RICARTE, Patrícia Chanely Silva. Um grito para o céu: arte e pensamento em Crônica da casa assassinada. 2007. 141 p. Dissertação (Mestrado em Estudos Literários) - Faculdade de Letras, Universidade Federal de Goiás, Goiânia, 2007.

ROSA E SILVA, Enaura Quixabeira. A equação da febre: uma leitura da Crônica da casa assassinada. 1994.92 p. Dissertação (Mestrado em Letras - Literatura Brasileira) - Centro de Ciências Humanas, Letras e Artes, Universidade Federal de Alagoas, Maceió, 1994.

- A condição humana na obra de Lúcio Cardoso: entre Eros e Tânatos, a alegoria barroca brasileira. 1999. 234 p. Tese (Doutorado em Estudos Lusobrasileiros) - Centro de Ciências Humanas, Letras e Artes, Universidade Federal de Alagoas; Université Stendhal-Grenoble 3, Maceió, AL; Paris, França, 1999.

ROSAS, Maria do Socorro. A ilustre casa dos Meneses: a narrativa em "Crônica da casa assassinada”. 1998. 215 p. Tese (Doutorado em Letras - Estudos Literários) Universidade Estadual Paulista Júlio de Mesquita Filho, UNESP, Araraquara, SP, 1998.

SANTOS, Cássia dos. Polêmica e controvérsia: o itinerário de Lúcio Cardoso de Maleita a O enfeitiçado. 1997. 201 p. Dissertação (Mestrado em Letras - Teoria Literária) - Instituto de Estudos da Linguagem, Universidade Estadual de Campinas, Campinas, 1997.

. Uma paisagem apocalíptica e sem remissão: a criação de Vila Velha e da

Crônica da casa assassinada. 2005. 282 p. Tese (Doutorado em Teoria e História Literária) - Instituto de Estudos da Linguagem, Universidade Estadual de Campinas, Campinas, 2005.

SANTOS, Odirlei Costa dos. Retratos do mal(-)estar no Diário completo, de Lúcio Cardoso. 2005. 97 p. Dissertação (Mestrado em Letras - Teoria da Literatura) - Universidade Federal de Juiz de Fora, Juiz de Fora, 2005.

SILVA, Denilson Lopes. Nós os mortos (sensibilidades melancólicas, imagens neo barrocas $[$ sic]). 1997. 283 p. Tese (Doutorado em Sociologia) - Universidade de Brasília, Brasília, 1997.

SILVA, Flávia Trocoli Xavier da. Fios da introspecção: para uma leitura do terceiro romance de Lúcio Cardoso. 2000. 143 p. Dissertação (Mestrado em Teoria e História Literária) - Universidade Estadual de Campinas, Campinas, 2000.

SILVA, Guilherme Ferreira. Formas de evasão em Lúcio Cardoso. 1972.93 p. Dissertação (Mestrado em Sociologia) - Faculdade de Filosofia, Letras e Ciências Humanas, Universidade de São Paulo, São Paulo, 1972. 
3/43/33/3/4. A cidade e a província em uma obra intimista. 1978. 173 p. Tese (Doutorado em Sociologia) - Faculdade de Filosofia, Letras e Ciências Humanas, Universidade de São Paulo, São Paulo, 1978.

SILVA, Maria Lucilene da. Desvios da angústia: uma leitura de O escravo, de Lúcio Cardoso. 2000. 100 p. Dissertação (Mestrado em Letras e Lingüística) - Centro de Ciências Humanas, Letras e Artes, Universidade Federal de Alagoas, Maceió, 2000.

SILVA, Miguel Furtado Freire da. Uma luz brasileira: A contribuição de Mario Carneiro. 2006. 150 p. Dissertação (Mestrado em Comunicação) - Universidade Federal Fluminense, Niterói, RJ, $2006{ }^{6}$

SILVA, Sandra Maria da. O jogo das metáforas pictórico-literárias na representação do ser integral: o expressionismo segundo Edvar Münch e Lúcio Cardoso. 2004. 89 p. Dissertação (Mestrado em Letras) - Universidade Federal do Pernambuco, Recife, PE, 2004. ${ }^{7}$

SILVA, Sérgio Luiz de Almeida. O diabo habita os lugares fechados. Teatro e intimidade em Lúcio Cardoso. 2003. 324 p. Dissertação (Mestrado em Teatro) - Universidade do Rio de Janeiro, Rio de Janeiro, 2003.

SOUZA JÚNIOR, José Luiz Foureaux de. Estética do romance intimista no Brasil. 1988. 104 p. Dissertação (Mestrado em Letras - Teoria da Literatura) Universidade de Brasília, Brasília, $1988 .^{8}$

. Caleidoscópio de vestígio e fragmentos: visóes da literatura intimista no Brasil. 1995. 244 p. Tese (Doutorado em Estudos Literários) - Universidade Federal de Minas Gerais, Belo Horizonte, $1995 .{ }^{9}$

${ }^{6}$ A dissertação aborda, entre outros assuntos, o filme Porto das caixas, cujo argumento é de Lúcio Cardoso.

${ }^{7}$ A autora fez, antes do mestrado, especialização em Letras com o trabalho: Alegoria da Liberdade na Polifonia de Lúcio Cardoso, apresentado à Universidade Federal de Pernambuco - UFPE, em 2001.

${ }^{8} \mathrm{O}$ escritor é citado às páginas 4, 17, 46-47, 55, 96, 98 e 100.

${ }^{9}$ Lúcio Cardoso comparece às páginas 10-12, 16, 44, 48, 71-72, 75-76, 79, 82-83, 85, $88,96,109,114,118,124-125,135,139-140,168-170,172,174,177-179,183-$ $184,214,217,230$ e 231. 
THOMÉ, Ricardo Luiz de Souza. Eros proibido: as ideologias em torno da questão homoerótica na literatura brasileira. 2002. 209 p. Tese (Doutorado em Literatura Brasileira) - Faculdade de Letras, Universidade Federal do Rio de Janeiro, Rio de Janeiro, 2002.

TOMAZ, Jerzuí Mendes Torres. Trilhamento do feminino: uma bordagem psicanalítica de Crônica da casa assassinada. 1999. 96 p. Dissertação (Mestrado em Letras e Lingüística) - Centro de Ciências Humanas, Letras e Artes, Universidade Federal de Alagoas, Maceió, 1999.

TROVÃO, Suzete Silva. Crônica da casa assassinada: uma reconstrução de vozes no terreno minado da transgressão. 2000. 3 v., 83 p. Dissertação (Mestrado em Letras) - Universidade do Estado do Rio de Janeiro, Rio de Janeiro, 2000.

VILELA, Andréa de Paula Xavier. Olhar sobre uma casa escrita - a poética de Lúcio Cardoso no romance Crônica da casa assassinada. 2002. 94 p. Dissertação (Mestrado Letras - Estudos Literários) - Faculdade de Letras, Universidade Federal de Minas Gerais, Belo Horizonte, 2002.

. Lúcio Cardoso - o traçado de uma vida. 2007. 204 p. Tese (Doutorado em Letras - Estudos Literários) - Faculdade de Letras, Universidade Federal de Minas Gerais, Belo Horizonte, 2007.

Como se pode notar, parte substancial desta bibliografia agrega trabalhos voltados ao estudo da Crônica da casa assassinada, romance publicado em 1959, considerado a obra-prima do autor. O restante se divide entre os livros Inácio, O enfeitiçado, Baltazar, Dias perdidos, Diário completo, A luz no subsolo, O escravo, o teatro como um todo, a poesia, a correspondência, principalmente a trocada com Clarice Lispector, a pintura, as polêmicas, o homoerotismo e o cinema.

Percebe-se, ainda, que a maioria dos estudos proveio da região Sudeste (26 dissertaçõos e 19 teses), vindo a seguir o Sul (sete dissertações e uma tese), o Centro-Oeste (cinco dissertações e uma tese) e o Nordeste (sete dissertações) do Brasil, além de duas teses defendidas na França. Destas, uma foi apresentada no sistema de co-tutela entre a Universidade Federal de Alagoas e a Université Stendhal-Grenoble 3, em Paris.

Dos 68 trabalhos, nove já se transformaram em livro e dois estão no prelo, dos seguintes autores: Cássia dos Santos (Mestrado), Enaura Quixabeira Rosa e Silva (Mestrado e Doutorado), Ésio Macedo Ribeiro (Mestrado, o Doutorado no prelo), Denílson Lopes (Doutorado), Maria 
Teresinha Martins (Doutorado), Mario Carelli (Doutorado), Marta Cavalcante de Barros (Mestrado), Ruth Silviano Brandão (Doutorado), Teresa de Almeida (Doutorado: no prelo). ${ }^{10}$

\begin{tabular}{llcccc}
\hline Universidade & Cidade & Estado & Dissertaçôes & Teses & Total \\
\hline UNESP & Araquara & SP & & 1 & 1 \\
UNESP & São J. R. Preto & SP & & 1 & 1 \\
USP & São Paulo & SP & 6 & 3 & 9 \\
UNICAMP & Campinas & SP & 3 & 1 & 4 \\
UFMG & Belo Horizonte & MG & 5 & 3 & 8 \\
CESJF & Juiz de Fora & MG & 1 & & 1 \\
UFJF & Juiz de Fora & MG & 1 & & 1 \\
URJ & Rio de Janeiro & RJ & 1 & & 1 \\
UFRJ & Rio de Janeiro & RJ & 3 & 9 & 12 \\
UERJ & Rio de Janeiro & RJ & 2 & & 2 \\
PUC & Rio de Janeiro & RJ & 2 & & 2 \\
UFF & Niterói & RJ & 1 & 1 & 2 \\
UFES & Vitória & ES & 1 & & 1 \\
UFG & Goiânia & GO & 4 & & 4 \\
UNB & Brasília & DF & 1 & 1 & 2 \\
FUFRG & Rio Grande & RS & 1 & & 1 \\
UFRGS & Porto Alegre & RS & 1 & 1 & 2 \\
UFPR & Curitiba & PR & 3 & & 3 \\
UFSC & Florianópolis & SC & 2 & & 2 \\
UFAL & Maceió & AL & 4 & & 4 \\
UFC & Fortaleza & CE & 2 & & 2 \\
UFPE & Recife & PE & 1 & & 1 \\
SORBONNE & Paris & França & & 1 & 1 \\
UFAL/ & & & & & \\
SORBONNE & Maceió/Paris & AL/Brasil & & 1 & 1 \\
& & França & & & \\
\hline TOTAL & & & 45 & 23 & 68 \\
\hline
\end{tabular}

${ }^{10}$ À frente do nome dos autores inseri, entre parênteses, a menção aos trabalhos publicados, se Mestrado ou Doutorado, ou ambos. Para maiores detalhes, conferir o item "Livros" da Parte 2 ("Bibliografia anotada (1934-2005)") do meu livro O riso escuro ou o pavão de luto: um percurso pela poesia de Lúcio Cardoso. RIBEIRO, 2006, p. 201-225. 
No gráfico a seguir, apresento as 45 dissertações de mestrado e as 23 teses de doutorado, a partir do ano de suas defesas:

\begin{tabular}{|c|c|c|c|}
\hline Ano & Dissertaçōes & Teses & Total \\
\hline 1972 & 1 & & 1 \\
\hline 1974 & 1 & & 1 \\
\hline 1978 & 1 & 1 & 2 \\
\hline 1980 & & 1 & 1 \\
\hline 1982 & & 1 & 1 \\
\hline 1985 & & 1 & 1 \\
\hline 1986 & & 1 & 1 \\
\hline 1987 & 1 & & 1 \\
\hline 1988 & 1 & & 1 \\
\hline 1989 & & 1 & 1 \\
\hline 1990 & & 1 & 1 \\
\hline 1991 & & 1 & 1 \\
\hline 1992 & 1 & & 1 \\
\hline 1993 & 1 & & 1 \\
\hline 1994 & 3 & & 3 \\
\hline 1995 & 1 & 1 & 2 \\
\hline 1997 & 4 & 1 & 5 \\
\hline 1998 & 1 & 1 & 2 \\
\hline 1999 & 1 & 1 & 2 \\
\hline 2000 & 6 & 1 & 7 \\
\hline 2001 & 1 & 1 & 2 \\
\hline 2002 & 2 & 3 & 5 \\
\hline 2003 & 4 & 1 & 5 \\
\hline 2004 & 3 & & 3 \\
\hline 2005 & 4 & 2 & 6 \\
\hline 2006 & 2 & 1 & 3 \\
\hline 2007 & 5 & 2 & 7 \\
\hline 2008 & 1 & & 1 \\
\hline TOTAL & 45 & 23 & 68 \\
\hline
\end{tabular}

Como se pôde notar, estudos universitários sobre a obra cardosiana começaram a aparecer timidamente para se avolumarem nos últimos dez anos, o que demonstra interesse cada vez maior pela obra de Lúcio Cardoso. Espero que este levantamento possa ser de alguma valia para os atuais e futuros estudiosos da obra cardosiana. 


\section{Referencias Bibliográficas}

RIBEIRO, Ésio Macedo. O riso escuro ou o pavão de luto: um percurso pela poesia de Lúcio Cardoso. Prefácio de Ruth Silviano Brandão, apresentação de Valentim Facioli. São Paulo: Edusp/Nankin Editorial, 2006.

. Bibliografia anotada (1934-2006). In: . Edição crítica da poesia reunida de Lúcio Cardoso. 2006. 2 v. 1.055 p. Tese (Doutorado em Letras Literatura Brasileira) - Faculdade de Filosofia, Letras e Ciências Humanas, Universidade de São Paulo, São Paulo, 2006. p. 739-826.

. Bibliografia anotada (1934-2008). In: CARDOSO, Lúcio. Poesia completa. Edição crítica de Ésio Macedo Ribeiro. Prefácio de João Adolfo Hansen. São Paulo: Edusp. [No prelo]

\section{Resumo}

Este texto apresenta a fortuna crítica (acadêmica) de Lúcio Cardoso, com o intuito de tornar conhecidos os trabalhos universitários que foram feitos sobre a obra do autor.

\section{Abstract}

This text presents the critical (academic) richness of Lúcio Cardoso, with the intention of making known the literary studies of the works of this author. 